\title{
Noise Spectrum of a Diode With a Retarding Field'
}

\author{
By Jacob J. Freeman
}

\begin{abstract}
A general expression for the noise-power spectrum generated by the random emission of electrons of arbitrary trajectories within a waveguide is obtained. A numerical solution of the potential distribution within a plane diode is effected, and a family of curves from which the potential distribution may be obtained is graphed. A graphical comparison between several actual potential distributions and Von Laue's solution is given. The above results are utilized to derive the equivalent mean-square fluctuation current due to the space charge within a diode for two cases of potential distribution, namely, the linear distribution, and that distribution which occurs at the neighborhood of the beginning of the retarding field. For the case of the linear potential distribution, the equivalent noise temperature of the diode conductance is shown to be equal to the cathode temperature.
\end{abstract}

\section{Introduction}

A considerable increase at high frequencies in both the shot noise and conductance of a negatively biased diode over the theoretical values computed on the basis of zero transit angle has been reported in the recent literature by several investigators. C. N. Smyth [1] ${ }^{2}$ observed excessive damping and noise from a negatively biased diode at $3,300 \mathrm{Mc} / \mathrm{s}$, and suggested it was due to currents induced by space charge. A. Van der Ziel [2] and A. Versnel [3] reported preliminary measurements on the equivalent noise current, and on the conductance of a negatively biased diode at wavelengths of 5.8 and $7.25 \mathrm{~m}$, over a range of anode voltages.

In this paper the noise-power spectrum of a diode with a retarding field ${ }^{3}$ will be investigated theoretically, taking into account only the noise power generated by those electrons with insufficient energy to reach the plate, in order to be able to evaluate the equivalent mean square current fluctuations due to space charge alone. ${ }^{4}$ Because

\footnotetext{
${ }^{1}$ A dissertation submitted to the faculty of the Graduate School of Arts and Sciences of the Catholic University of America in partial fulfillment of the requirements for the degree of Doctor of Philosophy.

${ }^{2}$ Figures in brackets indicate the literature references at the end of this paper.

${ }^{3} \mathrm{By}$ a retarding field is meant one in which the slope of the potential curve is always negative.

" For brevity, the term "space charge" will henceforth be used to designate the aggregate of electrons that have insufficient energy to reach the plate.
}

of the mathematical intractability of the potential distribution, the analysis is carried out only for the case of large saturation current, when the slope of the potential curve at the anode is small, and the case of small saturation current, when the potential distribution is essentially linear. For the latter case, the equivalent temperature of the conductance is shown to be equal to the cathode temperature.

Coincidentally, the noise power spectrum generated by electrons of prescribed trajectory within a waveguide of arbitrary cross section is developed, partly because the results can be immediately applied to the specific problem of a diode across a transmission line, and partly because the results are necessary for intended investigations of noise from transit-time devices within waveguides.

\section{Noise Spectrum}

In this section ${ }^{5}$ will be derived an expression for the noise power generated by random emission within a waveguide, or a transmission line. The latter is to be regarded as a special case of the former, in which the particular mode propagated is the TEM mode, and the word "guide" will be used to designate both. Although in this paper only the particular case of a retarding-field diode

\footnotetext{
${ }^{5}$ MKS units are employed throughout this paper.
} 
connected to a transmission line will be considered in detail, the noise spectrum of a diode inserted within a cylindrical guide of arbitrary cross section will be treated, since the generalization involves little further complication, and it seems desirable to have an explicit treatment of the problem for purposes of future reference (e. g., computation of the noise power generated by electronic transit-time devices within waveguides). It is assumed that the cathode and anode surfaces of the diode coincide with parts of the guide surface, so that there are no discontinuities due to the electrode structure. In other words, electrons emitted from a given portion of the guide surface describe a trajectory that is prescribed for electrons with given initial velocity by a given direct-current potential and impinge on another portion of the guide surface. It is assumed that the given direct-current potential has no minimum, so that the results of this section will apply either to the case of a temperature-saturated diode, or one with a retarding field, in which the anode voltage is sufficiently below the cathode, so that the slope of the potential curve at the anode is negative. If no direct-current potential minimum exists, the perturbations of the trajectory of electrons with a given initial velocity due to small fluctuations in the space-charge potential will be small, and it is assumed that they can be neglected. If a potential minimum exists (fig. 1), there is a discontinuous change in trajectory for electrons having just enough initial energy to reach the potential minimum, so that a small

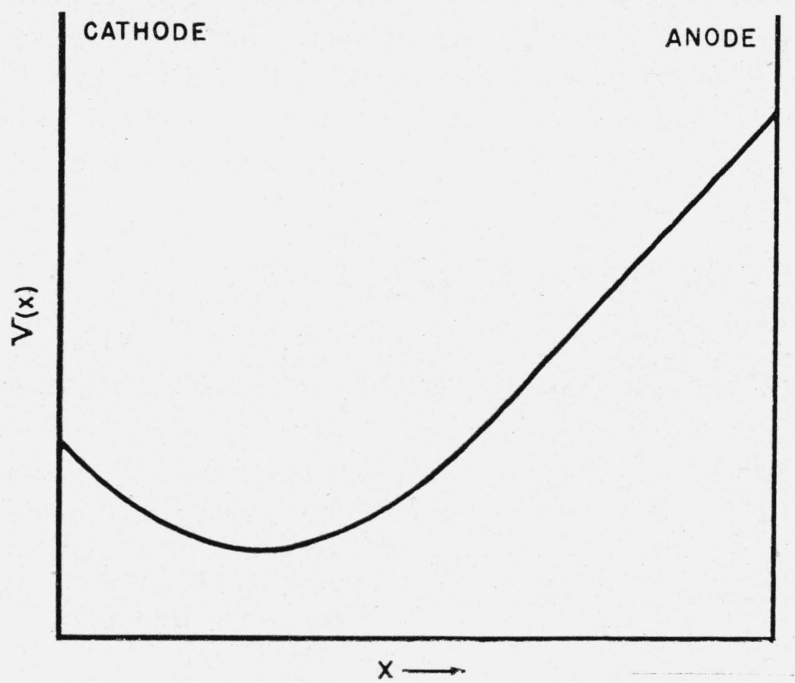

Figure 1. Voltage distribution of diode with minimum. fluctuation of potential may cause a large fluctuation of trajectory for this class of electrons.

Accordingly, then, it may reasonably be assumed that each electron with a given initial velocity traverses a fixed trajectory independently of all other electrons; thus, one may regard the resulting electromagnetic pulses generated by each electron as random events.

The guide is considered to have its axis in the $z$-direction, and to be terminated at either end by a matched load, with the diode in the center. The immediate problem is to compute the average noise energy absorbed by each load, due to electrons having an arbitrary trajectory. Only the case where the range in frequency is such that the guide supports only the dominant mode will be considered.

Then if $\boldsymbol{E}=\boldsymbol{E}_{0} \exp (j \omega t)$ represents the electric field of the dominant mode generated by a current density $\boldsymbol{J}=\boldsymbol{J}_{0} \exp (j \omega t)$, it is shown in appendix 1 that

$$
\boldsymbol{E}_{0}=-\frac{\mu \omega}{2 \alpha} \boldsymbol{b}(\boldsymbol{r}) \int \boldsymbol{J}\left(\boldsymbol{r}^{\prime}\right) \cdot \boldsymbol{b}\left(\boldsymbol{r}^{\prime}\right) \exp \left(-j \alpha\left|z^{\prime}-z\right|\right) d \tau^{\prime} .
$$

Here $\mu$ is the magnetic permeability, and $d \tau^{\prime}$ is an element of volume occupied by the current density. $\omega=2 \pi f$ is the angular frequency, $\alpha$ the phase constant for the dominant mode, and $\boldsymbol{b}$ is the corresponding normal mode [4]. Some properties of $\boldsymbol{b}$ are briefly discussed in appendix 1. $\boldsymbol{r}(x, y, z)$ and $\boldsymbol{r}^{\prime}\left(x^{\prime}, y^{\prime}, z^{\prime}\right)$ are the coordinate vectors of the field and source points, respectively. If the current density $\boldsymbol{J}\left(\boldsymbol{r}^{\prime}\right)$ has negligible cross section, $\boldsymbol{J}\left(\boldsymbol{r}^{\prime}\right)$ $d \tau^{\prime}=I\left(\boldsymbol{r}^{\prime}\right) \boldsymbol{d} \boldsymbol{s}^{\prime}$, where $I\left(\boldsymbol{r}^{\prime}\right)$ is the current flowing at $\left(x^{\prime}, y^{\prime}, z^{\prime}\right)$, and $\boldsymbol{d} \boldsymbol{s}^{\prime}$ is the incremental displacement along the path of current flow, $s^{\prime}$, corresponding to $d \tau^{\prime}$. Equation 1 then becomes

$$
\boldsymbol{E}_{0}(\boldsymbol{r})=-\frac{\mu \omega}{2 \alpha} \boldsymbol{b}(\boldsymbol{r}) \int I\left(\boldsymbol{r}^{\prime}\right) \boldsymbol{d} \boldsymbol{s}^{\prime} \cdot \boldsymbol{b}\left(\boldsymbol{r}^{\prime}\right) \exp \left[j \alpha\left(z^{\prime}-z\right)\right], z>z^{\prime}
$$

When the current $I\left(x^{\prime}, y^{\prime}, z^{\prime}\right)$ consists of an electron of charge $e$ following the trajectory $s^{\prime}$,

$$
I=e \delta\left(t-t^{\prime}\right)=\frac{e}{2 \pi} \int_{-\infty}^{\infty} \exp \left[j \omega\left(t-t^{\prime}\right)\right] d \omega,
$$

where $t^{\prime}$ represents the time at which the electron is at $\left(x^{\prime}, y^{\prime}, z^{\prime}\right)$, and $\delta\left(t-t^{\prime}\right)=0$, for $t \neq t^{\prime}$, and $\int \delta\left(t-t^{\prime}\right) d t=1$. Accordingly, the field generated by an electron is 


$$
\begin{aligned}
\boldsymbol{E}(t)= & -\frac{\mu e \boldsymbol{b}}{2} \int_{-\infty}^{\infty} \frac{d \omega}{2 \pi} \exp [j(\omega t-\alpha z)] \frac{\omega}{\alpha} \\
& \int_{0}^{t_{0}} \boldsymbol{v}\left(t^{\prime}\right) \cdot \boldsymbol{b} \exp \left[j\left(\alpha z^{\prime}-\omega t^{\prime}\right)\right] d t^{\prime},
\end{aligned}
$$

where the variable of integration is changed from $s^{\prime}$ to $t^{\prime}, \boldsymbol{v}\left(t^{\prime}\right)=d \boldsymbol{s}^{\prime} / d t^{\prime}$, and $t_{0}$ is the transit time of the electron.

Let

$$
s(\omega)=\int_{0}^{t_{0}} \boldsymbol{v}\left(t^{\prime}\right) \cdot \boldsymbol{b} \exp \left[j\left(\alpha z^{\prime}-\omega t^{\prime}\right)\right] d t^{\prime} .
$$

Then,

$$
\boldsymbol{E}(t)=-\frac{\mu e \boldsymbol{b}}{4 \pi} \int_{-\infty}^{\infty} \frac{\omega}{\alpha} \exp [j(\omega t-\alpha z)] s(\omega) d \omega .
$$

Since $\boldsymbol{H}=j / \mu \omega$ curl $\boldsymbol{E}$, one finds similarly that

$$
\begin{aligned}
\boldsymbol{H}=- & \frac{j e}{4 \pi} \int_{-\infty}^{\infty} \exp [j(\omega t-\alpha z)] s(\omega) d \omega \\
& {\left[\frac{-j \alpha \boldsymbol{k} \times \boldsymbol{b}+\operatorname{curl} \boldsymbol{b}}{\alpha}\right] . }
\end{aligned}
$$

Since it is desired that $\boldsymbol{E}(t)$ be real, $\alpha$ is defined as an odd function of $\omega$, so that the imaginary parts of the integrals in eq. 6 and 7 vanish, since they are odd functions of $\omega$.

The instantaneous power flow due to the passage of a single electron, is, by Poynting's Theorem,

$$
P(t)=\int_{S^{\prime}} \boldsymbol{E} \times \boldsymbol{H} \cdot \boldsymbol{n} d a,
$$

where $\boldsymbol{n}$ is a unit normal to the surface $S^{\prime}$.

If one utilizes the orthogonality properties of the normal modes, as stated in eq. 94 and 95 in appendix 1, namely,

$$
\int_{S^{\prime}} \boldsymbol{b}_{j} \cdot \boldsymbol{b}_{h} d a=\delta_{j h}
$$

and

$$
\int_{S^{\prime}}\left[\boldsymbol{b}_{j} \times\left(\nabla x \boldsymbol{b}_{h}\right] \cdot k d a=0\right.
$$

it is found on substituting eq 6 and 7 in eq 8 , that

$$
\begin{aligned}
P(t)= & \frac{\mu e^{2}}{16 \pi^{2}} \int_{-\infty}^{\infty} \frac{\omega}{\alpha} \exp (j(\omega t-\alpha z)) s(\omega) d \omega \\
& \int_{-\infty}^{\infty} \exp [j(\omega t-\alpha z)] s(\omega) d \omega .
\end{aligned}
$$

Since the pulses of energy are random, or statistically independent, the average power flow, by Campbell's Theorem [5], is

$$
\bar{P}=\overline{P(t)}=\lambda \int_{-\infty}^{\infty} P(t) d t,
$$

where $\lambda$ is the average number of electrons emitted per second, and $\lambda e=I$, the direct current.

$$
\begin{aligned}
\bar{P}= & \frac{\lambda e^{2} \mu}{16 \pi_{2}} \int_{-\infty}^{\infty} \int_{-\infty}^{\infty} \frac{\omega}{\alpha} \exp [j(\omega t-\alpha z)] s(\omega) d \omega \\
& \int_{-\infty}^{\infty} s(\omega) \exp [j(\omega t-\alpha z)] d \omega d t .
\end{aligned}
$$

By Parseval's Theorem [6],

$$
\int_{-\infty}^{\infty} f_{1}(t) f_{2}(\mp t) d t=\int_{-\infty}^{\infty} F_{2}(f) F_{1}( \pm f) d f,
$$

where $f_{1}$ and $f_{2}$ are the Fourier transforms of $F_{1}$ and $F_{2}$.

Substituting eq 11 in eq 10 ,

$$
\bar{P}=\frac{\lambda e^{2} \mu}{8 \pi} \int_{-\infty}^{\infty} \frac{\omega}{\alpha} s(\omega) s(-\omega) d \omega .
$$

Or, since $s(-\omega)=\mathrm{s}^{*}(\omega)$ where $s^{*}$ is the complex conjugate of $s$, and since $s^{*} s$ is even in $\omega$,

$$
\bar{P}=\frac{e I \mu}{4 \pi} \int_{0}^{\infty} \frac{\omega}{\alpha}|s(\omega)|^{2} d \omega .
$$

If one defines $\omega \mu / \alpha=Z$, the wave impedance, then

$$
\bar{P}=\frac{e I}{4 \pi} \int_{0}^{\infty} Z(\omega)|s(\omega)|^{2} d \omega .
$$

We consider now a plane parallel transmission line, with planes a distance $a$ apart, and of width $d$ (fig. 2). For this case, $\boldsymbol{b}=\boldsymbol{i} / \sqrt{a d}$, and $\alpha=\omega \sqrt{\mu \epsilon}$, where $\boldsymbol{i}$ is a unit vector in the $x$-direction, and $\boldsymbol{\epsilon}$ is the specific inductive capacity of free space. Hence,

$$
\overline{\boldsymbol{P}}=\frac{e I}{4 \pi} \sqrt{\mu \epsilon} \int_{0}^{\infty}|s(\omega)|^{2} d \omega
$$

where

$$
s(\omega)=\int_{0}^{t_{0}} \frac{\boldsymbol{v}\left(t^{\prime}\right) \cdot \boldsymbol{i} \exp j\left(\omega \sqrt{\mu \epsilon} z^{\prime}-\omega t^{\prime}\right)}{\sqrt{a d}} d t^{\prime}
$$

If the trajectory is considered to be in the $x, y$, plane, and the linear dimensions of the cathode small compared to the wavelength corresponding. to any frequency in the range considered, then one may take $z^{\prime}=0$ and let the origin of coordinates be at the cathode. Equation 15 becomes 


$$
s(\omega)=\int_{0}^{t_{0}} \frac{\boldsymbol{v}\left(t^{\prime}\right) \cdot \boldsymbol{i} \exp \left(-j \omega t^{\prime}\right) d t^{\prime}}{\sqrt{a d}}
$$

In order to compute the power spectrum from a diode with a retarding field the trajectories must be computed, and this requires that the potential distribution for the case of a retarding field be determined. This is indicated in the next section.

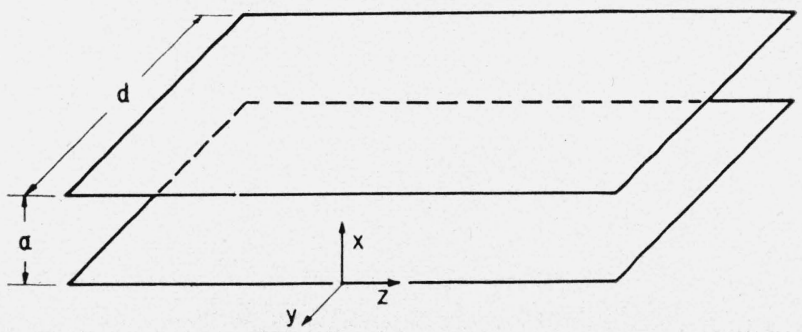

FIgURE 2. Schematic of parallel-plane transmission line.

\section{Potential Within a Plane Parallel Diode Subject to a Retarding Field}

\section{Rigorous Solution}

The potential distribution within a conducting diode has been thoroughly investigated by Fry [7] and Langmuir [8], among others. However, Fry limited his investigations to the case where the potential has a minimum. Von Laue [9] has exhaustively treated the potential distribution of a diode for the case of a retarding field, where it is assumed that the plate current, $I_{p}$, makes a negligible contribution to the potential in comparison to the contribution made by those electrons that never reach the anode. In other words, Von Laue assumes that the electron gas between the two electrodes is in statistical equilibrium, and that the space-charge distribution is governed by the familiar relation,

$$
\rho=\rho_{0} \exp \left(-\frac{e V}{k T}\right),
$$

where $\rho$ is the space charge per unit volume. Since the rigorous potential distribution for the case of a retarding field has never been investigated, it was believed desirable that this be done, in order to check the accuracy and range of Von Laue's solution.

The separation, $a$, between cathode and anode (fig. 3) is considered to be sufficiently small with respect to the area of the electrodes so that edge effects may be neglected, and the potential distribution is governed by

$$
\frac{d^{2} V}{d x^{2}}=-\frac{\rho}{\epsilon}
$$

where $V$ is the potential, $\rho$ the charge density, and

$$
\epsilon=1 / 36 \pi \cdot 10^{-9} \text { farad per meter. }
$$

The anode is assumed to be at a voltage $V_{1}$ sufficiently negative with respect to the cathode

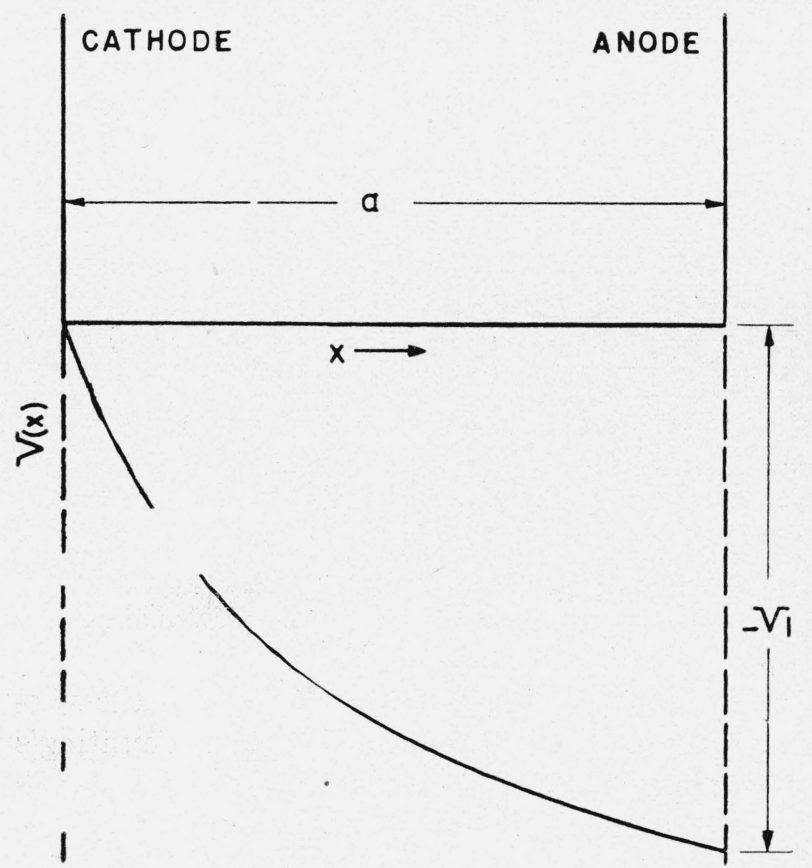

Figure 3. Voltage distribution of diode with retarding field.

so that the slope of the potential $V(x)$ is always negative.

The following analysis follows Fry's analysis of the $\alpha$-space precisely, and is outlined here for completeness. The only difference, of course, is that here the constant of integration is not zero, as it is when one has a potential minimum. If $v_{0}$ represents the initial velocity of an electron, and $v$ is its velocity at position $x$,

$$
\frac{1}{2} M v^{2}+e V=\frac{1}{2} M v_{0}^{2},
$$

where $e=-1.6010^{-19}$ coulomb, is the charge of the electron, and $M=9 \cdot 10^{-31}$, is the mass of the electron in kilograms.

At a given position $x$, those electrons having initial velocities such that $1 / 2 M v_{0}^{2}<e V$ never reach $x$; those having initial velocities such that $e V<1 / 2 M v_{0}^{2}<e V_{1}$ pass by $x$ twice, going and 
returning. Those having initial velocitis such that $1 / 2 M v_{0}^{2}>e V_{1}$ are captured by the plate and pass the point only once. Accordingly, the charge density is given by

$$
\rho(x)=e \int_{\sqrt{r V_{1}}}^{\infty} \frac{n\left(v_{0}\right)}{v} d v_{0}+2 e \int_{\sqrt{r V(x)}}^{\sqrt{r V_{1}}} \frac{n\left(v_{0}\right)}{v} d v_{0},
$$

where $n\left(v_{0}\right)$ is the number of electrons emitted per second per square meter with initial velocities between $v_{0}$ and $v_{0}+d v_{0}$, and $r=2 e / M$. Substituting eq 19 and 18 in eq 17 ,

$$
\frac{d^{2} V}{d x^{2}}=-\frac{e}{\epsilon}\left[\int_{\sqrt{r V_{1}}}^{\infty} \frac{n\left(v_{0}\right) d v_{0}}{\sqrt{v_{0}^{2}-r V}}+2 \int_{\sqrt{r V}}^{\sqrt{r V_{1}}} \frac{n\left(v_{0}\right) d v_{0}}{\sqrt{v_{0}^{2}-r V}}\right] .
$$

Integrating eq 20 between the limits $x$ and $a$, we get

$$
\begin{aligned}
\left(\frac{d V}{d x}\right)_{a}^{2}- & \left(\frac{d V}{d x}\right)^{2}= \\
& \frac{2 M}{\epsilon}\left[\int_{\sqrt{r V_{1}}}^{\infty} n\left(v_{0}\right) \sqrt{v_{0}^{2}-r V_{1}} d v_{0}-\right. \\
& \int_{\sqrt{r V_{1}}}^{\infty} n\left(v_{0}\right) \sqrt{v_{0}^{2}-r V} d v_{0}- \\
& \left.2 \int_{\sqrt{r V}}^{\sqrt{r V_{1}}} n\left(v_{0}\right) \sqrt{v_{0}^{2}-r V} d v_{0}\right] .
\end{aligned}
$$

Since the initial velocities are distributed according to the Maxwell-Boltzmann distribution,

$$
n\left(v_{0}\right)=\frac{N M}{k T} v_{0} \exp \left(-\frac{M v_{0}^{2}}{2 k T}\right)
$$

in which $N$ is the total number of electrons emitted per square meter per second, $k$ is Boltzmann's constant, $k=1.3810^{-23}$, and $T$ is the absolute temperature.

$$
I_{0}=N e,
$$

where $I_{0}$ is the saturation current density. If one changes to reduced variables,

and

$$
\xi=\sqrt{\zeta}(x-a),
$$

$$
\eta=\frac{e\left(V_{1}-V\right)}{k T},
$$

where

$$
\alpha=\frac{\sqrt{2 \pi M}}{\epsilon} \frac{e I_{p}}{(k T)^{3 / 2}},
$$

and $I_{p}$ is the plate current, then eq 21 reduces, when $n\left(v_{0}\right)$ is replaced by its value, (eq 22), to the following:

$$
\left(\frac{d \eta}{d \xi}\right)^{2}=\left(\frac{d \eta}{d \xi}\right)_{0}^{2}+e^{\eta}(1+\operatorname{erf} \sqrt{\eta})-\left(1+2 \frac{\sqrt{\eta}}{\sqrt{\pi}}\right) .
$$

Here erf $\eta$ is the error function,

$$
\operatorname{erf} x=\frac{2}{\sqrt{\pi}} \int_{0}^{x} \exp \left(-t^{2}\right) d t,
$$

and $(d \eta / d \xi)_{0}^{2}$ is the value of $(d \eta / d \xi)^{2}$ at $\xi=0$, that is, at the anode, $x=a$.

For brevity, let

$$
f(\eta)=(1+\operatorname{erf} \sqrt{\eta}) e^{\eta}-\left(1+\frac{2}{\sqrt{\pi}} \sqrt{\eta}\right) .
$$

and

$$
\left(\frac{d \eta}{d \xi}\right)_{0}=-m
$$

Accordingly, eq 26 may be written as

$$
\left(\frac{d \eta}{d \xi}\right)^{2}=m^{2}+f(\eta)
$$

Since $\xi$ is always negative,

$$
\xi=-\int_{0}^{\eta} \frac{d \eta}{\sqrt{f}(\eta)+m^{2}} .
$$

The value of $m$ is determined by the boundary condition at the cathode.

At the cathode, $x=0, \xi=-a \sqrt{\zeta}=\zeta_{1}, \eta=e V_{1} / k T=\eta_{1}$, so that

$$
\xi_{1}=-\int_{0}^{\eta_{1}} \frac{d \eta}{\sqrt{f(\eta)+m^{2}}}
$$

fixes the value of $m^{2}$.

Since for large values of $\eta, f(\eta) \rightarrow 2 e^{\eta}$, the upper limit of the integral in eq 32 may be replaced by infinity, so that one has, approximately,

$$
\xi=-\int_{0}^{\infty} \frac{d \eta}{\left(f(\eta)+m^{2}\right)^{\frac{1}{2}}} .
$$

Since $\xi_{1}$ is proportional to $I_{p}^{\frac{1}{2}}$, it may be inferred from the above equation that a large value of $m^{2}$ implies a small value of plate current, and that a small value of $m^{2}$ implies a relatively large value of plate current.

North's paper [10] tabulates $f(\eta)$ (his $\phi_{\alpha}(\eta)$ ), and tabulates the solution of eq 14, for the case $m^{2}=0$, (his $\xi_{\alpha}$ ). Using the computed values of 
$f(\eta)$ from North's paper, $\xi$ was determined as a function of $\eta$ by numerical integration, for selected values of $\mathrm{m}^{2}$, and the results are tabulated in table 1 for $0 \leqslant \eta \leqslant 20$, and are graphed in figure 4 for $0 \leqslant \eta \leqslant 10$. To determine $\xi$ for $\eta>9$, one observes that for the tabulated values of $\mathrm{m}^{2}$, $f(\eta)+m^{2} \simeq 2 e^{\eta}$, for $\eta>9$. Accordingly for $\eta>9$, eq 14 yields

$$
\xi(\eta)=\xi(9)+\sqrt{2}\left(e^{-4.5}-e^{-\eta / 2}\right),
$$

from which $\xi(\eta)$ may be calculated for larger values of $\eta$ than are tabulated in table 1 .

To determine the potential distribution of a given tube, one first determines $\xi_{1}$ and $\eta_{1}$ from the given values of cathode temperature, plate current, or saturation current, plate voltage, and plate-cathode separation, $a$. Then, from the family of $\xi, \eta$ curves plotted in figure 4, one determines the particular curve of the family that passes through the point $\xi_{1}, \eta_{1}$. From this curve $\eta(\xi),\left(\eta<\eta_{1}, \xi<\xi_{1}\right)$, the potential $V(x)$ within the diode may be determined from

$$
\begin{gathered}
\frac{V(x)}{V_{1}}=\frac{\eta_{1}-\eta}{\eta_{1}}, \\
\frac{x}{a}=\frac{\xi_{1}-\xi}{\xi_{1}},
\end{gathered}
$$

according to the definitions of $\xi$ and $\eta$ (eq 24 and $25)$.

\section{Approximate Analytic Expressions for the Potential}

In order to obtain an analytic approximation for the potential, one must examine approxima-

\begin{tabular}{|c|c|c|c|c|c|c|c|c|c|c|}
\hline$\eta$ & $m^{2}=0-\xi$ & $m^{2}=0.05-\xi$ & $m=0.1-\xi$ & $m^{2}=0.2-\xi$ & $m^{2}=0.3-\xi$ & $m^{2}=0.5-\xi$ & $m^{2}=1-\xi$ & $m^{2}=3-\xi$ & $m^{2}=10-\xi$ & $m^{2}=20-\xi$ \\
\hline 0 & 0 & 0 & 0 & 0 & 0 & 0 & 0 & 0 & 0 & 0 \\
\hline 0.01 & .......... & 0.0426 & 0.0309 & 0.0221 & 0.0181 & 0.0141 & & ......... & .......... & $\ldots$ \\
\hline .02 & ......... & .0814 & .0601 & .0437 & .0359 & .0280 & 0.0199 & 0.0115 & ......... & \\
\hline .03 & -....... & .1171 & .0880 & .0652 & .0533 & .0418 & ........ & ........ & ......... & \\
\hline .04 & ........ & .1503 & .1147 & .0858 & .0704 & .0554 & .0396 & .0230 & ....... & . \\
\hline .05 & 0.4281 & .1814 & .1402 & .1057 & .0873 & .0688 & ........... & -.......... & & ...... \\
\hline .06 & .......... & .2108 & .1648 & .1252 & .1038 & .0821 & .0590 & .0344 & & \\
\hline .07 & ........... & .2386 & .1884 & .1443 & .1200 & .0952 & -......... & & .... & \\
\hline .08 & ............ & .2650 & .2111 & .1628 & .1360 & .1082 & .0782 & .0458 & ............. & ........... \\
\hline .09 & ......... & .2902 & .2337 & .1808 & .1516 & .1210 & -......... & ......... & .......... & \\
\hline .10 & .5941 & .3141 & .2542 & .1984 & 16. 70 & .1337 & .0971 & .0572 & . & . \\
\hline .20 & .8170 & .5171 & .434 & .3565 & .3080 & .2528 & .1881 & .1130 & & \\
\hline .30 & .9785 & .6677 & .578 & .4887 & .4285 & .3590 & .2730 & .1673 & -... & \\
\hline .40 & 1. 1081 & .7918 & .697 & .6006 & .5339 & .4545 & .3521 & .2200 & & \\
\hline .50 & 1. 2173 & .9077 & .801 & .6985 & .6277 & .5409 & .4258 & .2710 & 0.1545 & 0.1105 \\
\hline .60 & 1. 3120 & .9903 & .891 & .7866 & .7116 & .6195 & .4945 & .3203 & ........ & ...... \\
\hline .70 & 1. 3956 & 1. 072 & .972 & .8638 & .7876 & .6914 & .5586 & .3678 & .......... & \\
\hline .80 & 1. 4704 & 1.146 & 1.045 & .9348 & .8568 & .7574 & .6184 & .4133 & ......... & ......... \\
\hline .90 & 1. 5380 & 1. 213 & 1. 111 & .9994 & .9201 & .8184 & .6744 & .4574 & -....... & -....... \\
\hline 1.0 & 1. 5996 & 1. 273 & 1. 171 & 1. 059 & .9786 & .8756 & .7269 & .4995 & .2997 & .2174 \\
\hline 2.0 & 2. 0134 & 1. 714 & 1.610 & 1. 488 & 1. 402 & 1. 302 & 1. 120 & .8355 & .5461 & .4106 \\
\hline 3.0 & 2. 2338 & 1. 933 & 1. 829 & 1. 701 & 1.615 & 1. $5 \Sigma 0$ & 1. 331 & 1.040 & .7259 & .5654 \\
\hline 4. 0 & 2. 3615 & 2. 059 & 1. 954 & 1.837 & 1.741 & 1. 647 & 1. 456 & 1. 164 & .8454 & .6762 \\
\hline 5.0 & 2. 4376 & 2. 134 & 2. 029 & 1.903 & 1.817 & 1. 722 & 1. 531 & 1. 239 & .9206 & .7490 \\
\hline 6.0 & 2. 4834 & 2. 179 & 2. 075 & 1. 947 & 1.864 & 1.769 & 1. 577 & 1. 282 & .9669 & .7946 \\
\hline 7.0 & 2. 5112 & 2. 206 & 2. 103 & 1. 976 & 1. 891 & 1. 795 & 1. 605 & 1. 312 & .9951 & .8227 \\
\hline 8 & 2. 5280 & 2. 224 & 2. 119 & 1. 992 & 1. 907 & 1.812 & 1. 621 & 1. $3 \varepsilon 9$ & 1. 0122 & .8398 \\
\hline 9 & 2. 5382 & 2. 234 & 2. 129 & 2. 002 & 1. 918 & 1.822 & 1. 631 & 1. 339 & 1. 0227 & .8502 \\
\hline 10 & 2. 5444 & 2. 2402 & 2. 1352 & 2. 0082 & 1. 9242 & 1.8282 & 1. 6372 & 1. 3452 & 1. 0289 & .8564 \\
\hline 12 & 2. 5504 & 2. 2462 & 2. 1412 & 2. 0142 & 1. 9302 & 1.8342 & 1. 6432 & 1. 3512 & 1. 0349 & .8624 \\
\hline 14 & 2. 5526 & 2. 2484 & 2. 1434 & 2. 0164 & 1. 9324 & 1. 8364 & 1. 6454 & 1. 3534 & 1. 0371 & .8646 \\
\hline 16 & 2. 5534 & 2. 2492 & 2. 1361 & 2. 0172 & 1. 9332 & 1. 8372 & 1. 6462 & 1. 3542 & 1. 0379 & .8654 \\
\hline 18 & 2. 5537 & 2. 2495 & 2. 1445 & 2. 0175 & 1. 9335 & 1.8375 & 1. 6465 & 1. 3545 & 1. 0382 & .8657 \\
\hline 20 & 2. 5338 & 2. 2496 & 2. 1446 & 2. 0176 & 1. 93366 & 1.8376 & 1. 6466 & 1. 3546 & 1. 0383 & .8658 \\
\hline
\end{tabular}
tions to $f(\eta)$ that will make the equation

TABLE 1. Values of $\xi=-\int_{0}^{\eta} \frac{d x}{\sqrt{f(x)+m^{2}}}$ as a function of $\eta$, for selected values of $m^{2}$ 


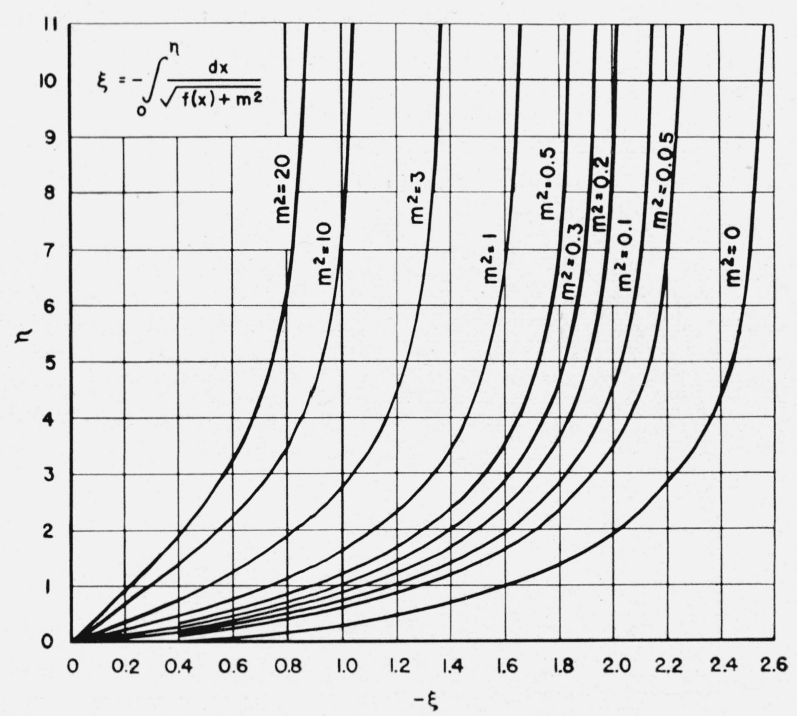

Figure 4. Family of $\xi, \eta$ curves.

$$
-\xi=\int_{0}^{\eta} \frac{d x}{\sqrt{f(x)+m^{2}}}
$$

integrable in terms of tabulated functions. For $\eta$ large, $f(\eta) \simeq 2 e^{\eta}$, and as $\eta \rightarrow 0, f(\eta) \rightarrow \eta$.

For $T=1,000^{\circ} K, \frac{e}{k T}=11.7$, and $\eta=11.7\left(V_{1}-V\right)$.

For negative plate voltages of one volt or more, $\eta$ will be larger than, say 2 , over most of the voltage range, so that the approximation $f(\eta) \simeq 2$ $\left(e^{\eta}-1\right)$ will be fairly good over most of the range of $\eta$. This is illustrated in table 2 , where $f(\eta)$ and $2\left(e^{\eta}-1\right)$ are tabulated for $0<\eta<9$. For

TABLE 2. A comparison of $f(\eta)$ and $2\left(e^{\eta}-1\right)$

\begin{tabular}{|c|c|c|}
\hline \multicolumn{1}{c|}{$|c| c \mid$} & $f(\eta)$ & $2(e \eta-1)$ \\
\cline { 1 - 2 } 0 & 0 & 0 \\
& & \\
0.2 & & \\
.4 & .294 & 0.44 \\
.6 & .716 & .98 \\
.8 & 1.27 & 1.64 \\
1 & 1.98 & 2.46 \\
& 2.88 & 3.44 \\
2 & & \\
3 & 11.8 & 12.78 \\
4 & 36.9 & 38.18 \\
5 & 105.6 & 107 \\
6 & 293 & 295 \\
& 803 & 804 \\
7 & 2,190 & 2,191 \\
8 & 5,960 & 5,960 \\
9 & 16,200 & 16.204 \\
\hline
\end{tabular}

large values of $\eta$ the approximation is quite good, but becomes poor for $0<\eta<2$. On the other hand, the error in $\xi$ due to the approximation is reduced by the addition of $m^{2}$, especially for larger values of $m^{2}$. Accordingly, let

$$
-\xi=\int_{0}^{\eta} \frac{d x}{\sqrt{2 e^{x}+m^{2}-2}}=\frac{1}{\sqrt{2}} \int_{0}^{\eta} \frac{d x}{\sqrt{e^{x}+A^{2}}},
$$

where

$$
A^{2}=\frac{m^{2}}{2}-1
$$

Equation 36 is a solution of

$$
\frac{d_{2} \eta}{d \xi^{2}}=e^{\eta},
$$

which is Von Laue's expression [9], inferred from the assumption that equilibrium exists. It is equivalent to

$$
\frac{d^{2} V}{d x^{2}}=-\frac{I_{0} \sqrt{\frac{2 \pi M}{k T}}}{\epsilon} e^{-\frac{e V}{k T}},
$$

which in turn implies that the space charge distribution is

$$
\rho=\rho_{0} e^{-\frac{e V}{k T}},
$$

where

$$
\rho_{0}=I_{0} \sqrt{\frac{2 \pi M}{k T}} .
$$

If one integrates the rigorous expression for $\rho$ in eq 19 , one gets

$$
\rho=I_{0} \sqrt{\frac{2 \pi M}{k T}} e^{-\frac{e V}{k T}\left(1+\operatorname{erf} \sqrt{\left.\frac{e\left(V_{1}-V\right)}{k T}\right)}\right.} .
$$

Since

$$
\operatorname{erf} \sqrt{\frac{e}{k T}\left(V_{1}-V\right)}=\operatorname{erf} \sqrt{\eta} \approx 1,
$$

for $\eta>2$, it is seen that the approximate expression for $\rho$ approaches the rigorous expression fairly closely for $\eta>2$, and accordingly, the approximate expression for the potential must also approach the true potential in this region.

For $-1 \leqslant A^{2}<0, \quad\left(0 \leqslant m^{2}<2\right)$, the integral of eq 36 is given by 


$$
\begin{aligned}
&-\xi= \frac{\sqrt{2}}{\sqrt{1-\frac{m^{2}}{2}}}\left[\cos ^{-1}\left(\sqrt{1-\frac{m^{2}}{2}} e^{-\eta / 2}\right)-\right. \\
&\left.\cos ^{-1} \sqrt{1-\frac{m^{2}}{2}}\right] .
\end{aligned}
$$

For $A^{2}=0,\left(m^{2}=2\right)$,

$$
\xi-\xi_{1}=\sqrt{2}\left(e^{-\eta / 2}-e^{-\eta_{1} / 2}\right) .
$$

For $A^{2}>0,\left(m^{2}>2\right)$,

$$
\begin{aligned}
-\xi= & \frac{\sqrt{2}}{\sqrt{\frac{m^{2}}{2}-1}}\left[\sinh ^{-1} \sqrt{\frac{m^{2}}{2}-1}-\right. \\
& \left.\sinh ^{-1}\left(e^{-\eta / 2} \sqrt{\frac{m^{2}}{2}-1}\right)\right] .
\end{aligned}
$$

'The value of $m^{2}$ in the above equations is fixed by the boundary conditions at the cathode, namely, $\eta=\eta_{1}$ when $\xi=\xi_{1}$. For large values of $m$, eq 45 gives

$$
m=-\frac{\eta_{1}}{\xi_{1}}
$$

and the resulting potential distribution is, of course, the same linear function of distance one gets in the absence of space charge.

In order to compare eq 43,44 , and 45 , which are Von Laue's solutions, with the rigorous solutions of eq 32 obtained numerically, values of the two solutions are tabulated in table 3 , for $\eta_{1}=10$, and for $m^{2}=0$, and $m^{2}=3$. For $m^{2}=3$, the agreement between the two solutions is excellent, and even for $m^{2}=0$, the worst possible case for a given value of $\eta_{1}$, the agreement is fair.

Unfortunately, the expressions for the potential obtained from eq 43 and 45 do not permit the time of transit of an electron as a function of its position to be calculated explicitly, which is necessary for an evaluation of the noise power spectrum.

However, the potential distribution given by eq 44 approximates fairly well the shape of the potential curve for small $m^{2}$, that is, $0<m^{2}<20$ (large $I_{0}$ ). In figure 5 graphs are plotted of the true reduced potential distribution, $V / V_{1}=$ $\left(\eta_{1}-\eta\right) / \eta_{1}$ as a function of the reduced distance $x / a=\left(\xi_{1}-\xi\right) / \xi_{1}$, for $m^{2}=0, m^{2}=10$, and $m^{2}=20$, for $\eta_{1}=10$. Also, the relative potential distribution obtained from eq 44 is plotted and is seen to fall about midway between the curves $m^{2}=0$ and $m^{2}=20$. Accordingly, for the case of large plate

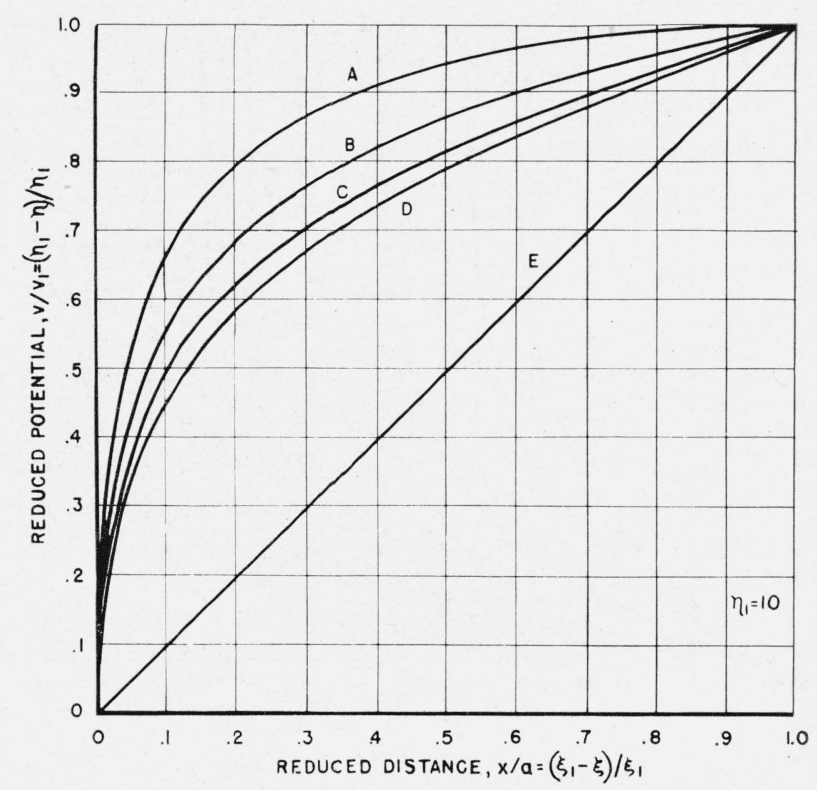

Figure 5. Comparison between approximate and rigorous potential distributions.

A, $m^{2}=0$; B, approximate formula; C, $m^{2}=10$; D, $m^{2}=20$; E, $m=-\eta_{1} / \xi_{1}$.

currents, one may use eq 44 for obtaining an approximate potential distribution and thence cal-

\begin{tabular}{|c|c|c|c|c|}
\hline \multicolumn{3}{|c|}{$m^{2}=0$} & \multicolumn{2}{|c|}{$m^{2}=3$} \\
\hline & Rigorous & $\begin{array}{l}\text { Approxi- } \\
\text { mate }\end{array}$ & Rigorous & $\begin{array}{l}\text { Approxi- } \\
\text { mate }\end{array}$ \\
\hline $1-\eta / \eta_{1}$ & $1-\xi / \xi_{1}$ & $1-\xi / \xi_{1}$ & $1-\xi / \xi_{1}$ & $1-\xi / \xi_{1}$ \\
\hline 0 & 0 & 0 & 0 & 0 \\
\hline 0.1 & 0.0025 & 0.0015 & 0.0045 & 0.0048 \\
\hline .2 & .0064 & .0061 & .0120 & .0125 \\
\hline .3 & .0130 & .0138 & .0247 & .0254 \\
\hline .4 & .0240 & .0264 & .0470 & .0466 \\
\hline .5 & .0420 & .0470 & .0789 & .0814 \\
\hline .6 & 0719 & .0814 & .135 & .139 \\
\hline .7 & .1221 & .1385 & .227 & .233 \\
\hline .8 & .209 & .234 & .379 & .386 \\
\hline .9 & .371 & .412 & .629 & .630 \\
\hline .95 & .522 & .566 & .799 & .798 \\
\hline .98 & .679 & .719 & .916 & .915 \\
\hline 1.0 & 1.000 & 1. 000 & 1.000 & 1.000 \\
\hline
\end{tabular}
culating the noise spectrum.

TABLE 3. A comparison of the reduced potential, $V / V_{1}:=1-\eta / \eta_{1}$, as a function of the reduced distance, $x / a=1-\xi / \xi_{1}$, obtained from the rigorous solution (eq 14), and from Von Laue's apprcximation (eq 43, 45)

For the case of small plate currents, and large negative anode voltages, the reduced potential distribution approaches a straight line of unit 
slope, also graphed in figure 5 under the label $m=-\eta_{1} / \xi_{1}$. Accordingly, the following section proceeds with the calculation of the noise-power spectrum for the two cases, large plate current ( $m^{2}$ small) and small plate current ( $m^{2}$ large).

\section{Noise Power Spectrum for a Diode}

\section{Case 1: Noise Power Spectrum for Large Plate Current ( $m^{2}$ small)}

If one substitutes the values of $\xi$ and $\eta$ from eq 34 and 35 into eq 44, one gets for the potential energy of an electron, $U$,

$$
U=V e=2 k T \ln (1+b x),
$$

where

$$
b=\frac{e^{\frac{e V_{1}}{2 \mathrm{k} T}}-1}{a} \simeq \frac{e^{\frac{e V_{1}}{2 \mathrm{k} T}}}{a} .
$$

In this paper only the contribution to the noise spectrum made by those electrons that never reach the plate, but which return to the cathode will be considered, since the effect of those electrons which constitute the plate current has been treated by Rack [11]. Accordingly, one considers only electrons whose initial kinetic energy, $E$, is less than $e V_{1}$.

For an electron emitted at $t=0$, the time at which it has position $x$ is given by

$$
t=\int_{0}^{x} \frac{d x}{\sqrt{2 / M} \sqrt{E-U(x)}} .
$$

If one substitutes for $U(x)$ its value given by eq 47 and integrates

$$
\begin{aligned}
t= & \sqrt{\frac{\pi M}{k T}} \frac{\exp (E / 2 k T}{2 b}\left[\operatorname{erf} \sqrt{\frac{E}{2 k T}}-\right. \\
& \left.\operatorname{erf} \sqrt{\frac{E}{2 k T}}-\ln (1+b x)\right]
\end{aligned}
$$

for $0 \leqslant t \leqslant t_{0 / 2}$, where $t_{0}$ is the transit time of the electron.

$$
t_{0}=\sqrt{\frac{\pi M}{k T}} \frac{\exp \left(\frac{E}{k T}\right)}{b} \operatorname{erf} \sqrt{\frac{E}{2 k T}}
$$

so that

$$
t=\frac{t_{0}}{2}\left(1-\frac{\operatorname{erf} \sqrt{\frac{E}{2 k T}-\ln (1+b x)}}{\operatorname{erf} \sqrt{\frac{E}{2 k T}}}\right)
$$

for $0 \leqslant t \leqslant t_{0} / 2$.

According to eq 14 and 16, the noise power, $P_{E}$, absorbed by each load in the frequency range $\Delta f$, due to electrons with initial energy $E$, is

where

$$
P_{E}=\frac{e I_{E}}{2} \sqrt{\frac{\mu}{\epsilon}}|s(\omega)|^{2} \frac{\Delta \omega}{2 \pi},
$$

$$
s(\omega)=\int_{0}^{t_{0} v \cdot t \exp (-j \omega t) d t} \frac{\sqrt{a d}}{\sqrt{a d}}
$$

and where $I_{E}$ is that portion of the saturation current composed of electrons of energy $E$.

Since $t(x)$ and $t^{\prime}(x)$, the first and second times at which the electron passes the point $x$, respectively, are related by

and since

$$
t^{\prime}=t_{0}-t
$$

$$
\begin{gathered}
v\left(t_{0}-t\right)=-v(t) \\
s(\omega)=\frac{1}{\sqrt{a d}}\left\{\int_{0}^{t_{0} / 2} v(t) \exp (-j \omega t) d t-\right. \\
\left.\int_{0}^{t_{0} / 2} v(t) \exp \left[j \omega\left(t-t_{0}\right)\right] d t\right\} .
\end{gathered}
$$

Letting$$
s_{1}(\omega)=\int_{0}^{t_{0} / 2} v(t) \exp (-j \omega t) d t=\int_{0}^{x_{m}} \exp (-j \omega t) d x
$$

where $x_{m}$ is the maximum value of $x$,

$$
s(\omega)=\frac{1}{\sqrt{a d}}\left[s_{1}(\omega)-\exp \left(-j \omega t_{0}\right) s_{1}(-\omega)\right] .
$$

Substituting eq 52 in eq 57 and 58 ,

$$
s(\omega)=\frac{2 i \exp \left(\frac{-j \omega t_{0}}{2}\right)}{\sqrt{a d}} \int_{0}^{x_{m}} \sin \left\{\frac{\omega t_{0} \operatorname{erf} \sqrt{\frac{E}{2 k T}-\ln (1+b x)}}{2 \operatorname{erf} \sqrt{\frac{E}{2 k T}}}\right\} d x
$$


To simplify the above somewhat, the substitution

$$
y=\sqrt{\frac{E}{2 k T}-\ln (1+b x)},
$$

is made, and one obtains

$$
s(\omega)=\frac{4 i \exp \left(\frac{E}{2 k T}-\frac{j \omega t_{0}}{2}\right)}{b \sqrt{a d}} \int_{0}^{\sqrt{\frac{E}{2 k T}}} y e^{-y^{2}} \sin \left\{\frac{\omega t_{0} \operatorname{erf} y}{2 \operatorname{erf} \sqrt{\frac{E}{2 k T}}}\right\} d y .
$$

Let

$$
A\left(\sqrt{\frac{E}{2 k T}}\right)=\int_{0}^{\sqrt{\frac{E}{2 k T}}} y e^{-y^{2}} \sin \left\{\frac{\omega t_{0} \operatorname{erf} y}{2 \operatorname{erf} \sqrt{\frac{E}{2 k T}}}\right\} d y .
$$

Then

$$
|s(\omega)|^{2}=\frac{16 a}{d} \exp \left(\frac{E-e V_{1}}{k T}\right) A^{2}\left(\sqrt{\frac{E}{2 k T}}\right),
$$

where the value for $b$ given by eq 48 has been substituted.

Since the energy distribution of the electrons is Maxwellian,

$$
I_{E}=\frac{I_{0}}{k T} \exp (-E / k T) d E,
$$

and since the resultant noise power due to uncorrelated noise sources is simply the sum of the powers of the individual sources [5], the resultant noise power spectrum due to all the electrons that return to the cathode is

$$
P=\frac{8 e I_{P}}{k T} \frac{a}{d} \sqrt{\frac{\mu}{\epsilon}} \int_{0}^{V_{1} e} A^{2}\left(\sqrt{\frac{E}{2 k T}}\right) d E \Delta f,
$$

where $P$ represents the noise power absorbed by each matched load in the interval $\Delta f=\Delta \omega / 2 \pi$.
The diode shunted by two matched transmission lines is now replaced by its equivalent circuit, namely, a diode shunted by impedance $Z_{0} / 2$. The equivalent mean square fluctuation current, $i^{\overline{2}}$ is defined by

$$
2 P=Z_{0} / 2 i^{2},
$$

where $Z_{0}=\sqrt{\mu / \epsilon} a / d$ is the characteristic impedance of the line. If the impedance of the diode is large compared to $Z_{0}$, which is assumed to be true, $i^{\overline{2}}$ represents the equivalent mean square fluctuation current that would flow through a diode shortcircuited at frequency $f$.

From eq 65 and 66 ,

$$
\overline{i^{2}}=\frac{32 e I_{P}}{k T} F\left(V_{1}, f\right) \Delta f
$$

where

$$
F\left(V_{1}, f\right)=\int_{0}^{e V_{1}} A^{2}(\sqrt{E / 2 k T}) d E
$$

Since

$$
\frac{\omega t_{0}}{2 \operatorname{erf} \sqrt{E / 2 k T}}=a \omega \sqrt{\pi M / 4 k T} \exp \left(\frac{E-e V_{1}}{2 k T}\right)
$$

from eq 48 and 51, letting

$$
c=a \omega \sqrt{\pi M / 4 k T},
$$

$$
A(\sqrt{E / 2 k T})=\int_{0}^{\sqrt{E / 2 k T}} u e^{-u 2} \sin \left\{c \exp \left(\frac{E-e V_{1}}{2 k T}\right) \operatorname{erf} u\right\} d u
$$

Integrating the above equation by parts,

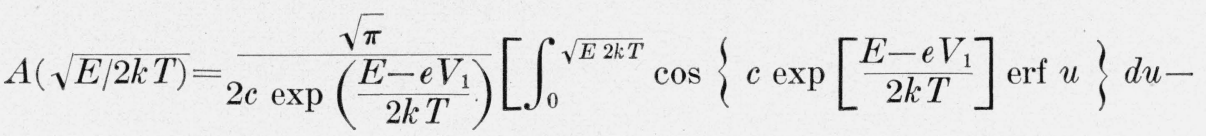

$$
\begin{aligned}
& \left.\sqrt{E / 2 k T} \cos \left\{c \exp \left[\frac{E-e V_{1}}{2 k T}\right] \text { erf } \sqrt{E / 2 k T}\right\}\right] \text {. }
\end{aligned}
$$


One observes that $A(\sqrt{E / 2 k T})=0$, for $c=0$ and $c=\infty$. Since the expression within brackets is always less than $2 \sqrt{E / 2 k T}$ in absolute value,

$$
A(\sqrt{E / 2 k T})<\frac{\sqrt{\pi} \sqrt{E / 2 k T}}{c \exp \left[\frac{E-e V_{1}}{2 k T}\right]}
$$

and

$F\left(V_{1}, f\right)=\int_{0}^{V_{1} e} A^{2}(\sqrt{E / 2 k T}) d E<\frac{\pi k T}{2 c^{2} \exp \left(-e V_{1} / k T\right)}$,

which gives an upper limit to $F\left(V_{1}, f\right)$ for very high frequencies.

$$
A(\sqrt{E / 2 k T})=\frac{c \exp \left(-\frac{e V_{1}}{2 k T}\right)}{2}\left[\frac{\exp (E / 2 k T)}{\sqrt{2}} \operatorname{erf} \sqrt{E / 2 k T}-\operatorname{erf} \sqrt{E / 2 k T}\right] .
$$

Since $A^{2}(\sqrt{E / 2 k T})$ approaches zero as $E / k T$ for $E / k T$ small, and increases exponentially as $\exp (E / k T)$ for $E / k T>1$, and since one is interested in $\int_{0}^{e V_{1}} A^{2} d E$ for an upper limit $E / k T=e V_{1} / k T>10$, the contribution to the integral from large values of the argument far overshadows the contribution from small values of the argument. Hence, we may replace the value of $A(\sqrt{E / 2 k T})$ by its asypmtotic value for large values of the argument,

$$
A(\sqrt{E / 2 k T})=\frac{c}{2 \sqrt{2}} \exp \left(\frac{E-e V_{1}}{2 k T}\right)
$$

Physically, this means that despite their preponderance in numbers, the low-energy electrons, because of their small excursions toward the plate and consequent short transit times, contribute practically nothing toward the low-frequency noise spectrum.

Accordingly,

where

$$
\begin{aligned}
& \int_{0}^{e V_{1}} A^{2} d E=\frac{k T c^{2}}{8}, \begin{array}{l}
\text { finds for the noise power } \\
\text { in the frequency interval }
\end{array} \\
& P=\frac{I_{0} e Z_{0}}{2 k T}\left(4 / e V_{1}\right)^{2} \int_{0}^{e V_{1}} E^{2} e^{-\frac{E}{k T}}\left\{\frac{\cos \sigma \sqrt{E}}{\sigma \sqrt{E}}-\frac{\sin \sigma \sqrt{E}}{\sigma^{2} E}\right\}^{2} d E \Delta f,
\end{aligned}
$$

$$
\sigma=\omega \sqrt{2 M} a / e V_{1} .
$$

The equivalent mean square fluctuation current through the diode is

$$
\overline{i^{2}}=\frac{2 I_{0} e}{k T}\left(4 / e V_{1}\right)^{2} \int_{0}^{e V_{1}} E^{2} e^{-\frac{E}{k T}}\left\{\frac{\cos \sigma \sqrt{E}}{\sigma \sqrt{E}}-\frac{\sin \sigma \sqrt{E}}{\sigma^{2} E}\right\}^{2} d E \Delta f .
$$


For low enough frequencies, such that $\sigma \sqrt{e V_{1}}<0.3$, the expression in brackets approximately equals $\sigma^{2} E / 9$. Since also,

$$
\int_{0}^{e V_{1}} E^{3} e^{-\frac{E}{k T}} d E \simeq 6(k T)^{4}, \text { for } e V_{1} / k T>10,
$$

$$
\overline{i^{2}}=\frac{128 M a^{2} \omega^{2} e I_{0}}{3\left(\frac{e V_{1}}{k T}\right)^{4} k T} \Delta f
$$

For large values of $V_{1}$, or for high frequencies, the upper limit in eq 80 may be taken as infinity with negligible error. Accordingly, eq 80 may be rewritten as

$$
\overline{i^{2}}=\frac{2 I_{0} e}{k T}\left(\frac{4}{e V_{1}}\right)^{2} \int_{0}^{\infty} E^{2} e^{\frac{-E}{k T}}\left\{\frac{\cos \sigma \sqrt{E}}{\sigma \sqrt{E}}-\frac{\sin \sigma \sqrt{E}}{\sigma^{2} E}\right\}^{2} d E \Delta f .
$$

In appendix 2 it is shown that

$$
\int_{0}^{\infty} E^{2} e^{\frac{-E}{k T}}\left\{\frac{\cos \sigma \sqrt{E}}{\sigma \sqrt{E}}-\frac{\sin \sigma \sqrt{E}}{\sigma^{2} E}\right\}^{2} d E=\frac{(k T)^{3}}{2}\left\{(1+2 w) e^{-w} F\left(\frac{1}{2}, \frac{3}{2}, w\right)-1\right\},
$$

where

$$
T_{e q}=T,
$$

$$
w=\sigma^{2} k T,
$$

and $F$ is Kummer's function [12], also known as the confluent hypergeometric function.

Accordingly, the expression for $\overline{i^{2}}$ may be written,

$$
\overline{i^{2}}=\frac{16 I_{0} e}{\left(\frac{e V_{1}}{k T}\right)^{2}}\left\{(1+2 w) e^{-w} F\left(\frac{1}{2}, \frac{3}{2}, w\right)-1\right\} \Delta f .
$$

\section{Equivalent Temperature of the Con- ductance Due to Space-Charge}

The shunt conductance, $g$, of the space-charge within a diode has recently been calculated by Begovich [13], who finds ${ }^{6}$

$$
g=\frac{4 I_{0} e}{k T\left(\frac{e V_{1}}{k T}\right)^{2}}\left\{(1+2 w) e^{-w} F\left(\frac{1}{2}, \frac{3}{2}, w\right)-1\right\},
$$

where $w$ is defined above (eq 85) and $F\left(\frac{1}{2}, \frac{3}{2}, w\right)$ is

the confluent hypergeometric function [12].

If the above value of $g$, and the value of $\overline{i^{2}}$ given by eq 86 are substituted in the Nyquist formula [24],

$$
\overline{i^{2}}=4 k T_{e q} g \Delta f,
$$

where $T_{\text {eq }}$ is the equivalent temperature of the conductance, it is immediately found that

\footnotetext{
${ }^{6}$ The writer is indebted to N. Begovich for a personal communication amplifying the material contained in reference [13].
}

in agreement with the experimental results obtained by Van der Ziel [3].

\section{Summary}

1. A general expression for the noise power spectrum generated by the random emission of electrons of arbitrary trajectories within a waveguide is developed in section $\mathrm{I}$.

2. A numerical solution of the potential distribution within a plane diode is effected in section II, and a family of curves from which the potential distribution may be obtained is graphed in figure 4. A graphical comparison between several actual potential distributions and their approximate representation is given in figure 5 .

3 . The results of sections I and II are utilized to derive the equivalent mean square fluctuation current due to the space charge within a diode for two types of a retarding potential distribution, namely, the linear distribution, and those distributions whose slope at the plate is small.

4. Utilizing the results of section III, and a recently computed value of the conductance due to the space charge within a diode, the equivalent noise temperature is shown to be equal to the cathode temperature.

The author is greatly indebted to K. F. Herzfeld for his many helpful discussions and generous guidance during the course of this work. Also, the writer is extremely grateful to H. Lyons, chief 
of the Microwave Standards Section, National Bureau of Standards, for his wholehearted cooperation and valuable criticisms.

\section{References}

[1] C. N. Smyth, Nature 15;, 841 (1946).

[2] A. Van der Ziel, Nature 159, 675 (1947).

[3] A. Versnel and A. Van der Ziel, Nature 159, 640 (1947).

[4] For properties and applications of normal modes to waveguides, see $\mathrm{H}$. Bethe, Formal theory of waveguides of arbitrary cross section, Radiation Laboratory Report 43-26 (Mar. 16, 1943).

[5] N. R. Campbell and V. J. Francis, J. Inst. Elec. Eng. 93, 45, (1946).

[6] G. Campbell and R. Foster, Fourier integrals for practical applications, Bell Telephone System Monograph B--584, p. 39 (1931).

[7] T. C. Fry, Phys. Rev. 17, 441 (1921).

[8] I. Langmuir, Phys. Rev. 21, 419 (1923).

[9] M. Von Laue, Jahrbuch der Radioaktivitat .und Electronik 15, 204 (1918).

[10] B. J. Thompson, D. O. North, W. A. Harris, RCA Rev. 4, 447 (Apr., 1940).

[11] A. J. Rack, Bell System Tech. J. 17, 592 (Oct. 1938).

[12] T. M. MacRobert, Functions of a complex variable", p. 346, 3d ed. (1947).

[13] N. Begovich, High frequency loading, Bull. Am. Phys. Soc. 23, No. 5 (June 24, 1948).

[14] H. Nyquist, Thermal agitation of electric charge in conductors, Phys. Rev. 32, 110 (1928).

[15] J. W. Miles, The equivalent circuit for a plane discontinuity in a cylindrical waveguide, Inst. Radio Eng. 34, 728 (Oct. 1946).

[16] T. M. MacRobert, Functions of a complex variable, 3d ed., p. 145 (Macmillian \& Co., London, 1947).

\section{Appendix 1}

Let $x, y$ be generalized coordinates suitable for describing the guide surface $S$, and $z$ the usual cartesian coordinate which is parallel to the axis of the guide. The normal modes, $\boldsymbol{b}_{j}$ of electric type for a cylindrical guide are a doubly infinite set obtained by two independent processes $[15,4]$.

$$
\boldsymbol{b}_{j}=\nabla U_{j} \times \boldsymbol{k}
$$

where $\boldsymbol{k}$ is a unit vector in the $z$-direction, and where

$$
\nabla^{2} U_{j}(x, y)+\beta_{i}^{2} U_{j}(x, y)=0,
$$

and $\boldsymbol{n} \cdot \nabla U_{j}=0$ on $\mathrm{S}$, the surface of the guide. is normal to $\mathrm{S}$.

$$
\boldsymbol{b}_{j}=\beta_{j}^{2} v_{j}(x, y) \boldsymbol{k} \mp j \alpha \nabla v_{j}(x, y),
$$

${ }_{7}$ The $v_{j}$ should not be confused with the letter $v$ used in section I to represent velocity. where $\nabla^{2} v_{j}+\beta_{j}^{2} v_{j}=0$, and $v_{j}=0$ on $S$, except for the TEM case for which $\beta_{j}=0$, and

$$
\boldsymbol{n} \times \nabla v_{3}=0 \text { on } S \text {. }
$$

(The $\beta_{j}$ are, in general, different for the $U_{j}$ and $v_{j}$.)

If the $U_{j}$ and $v_{j}$ are normalized, that is,

$$
\int_{S^{\prime}} U_{j}^{2} d a=\int_{S} v_{j}^{2} d a=1,
$$

where the integral is taken over the transverse plane $S^{\prime}$ of the guide, then the following orthogonal properties may be demonstrated $[15,4]$ :

$$
\int_{S^{\prime}} \boldsymbol{b}_{j} \cdot \boldsymbol{b}_{h} d a=\delta_{j h},
$$

where $\delta_{j h}=0$, for $j \neq h$, and $\delta_{j h}=1$, for $j=h$.

$$
\int_{S^{\prime}}\left(\boldsymbol{b}_{i} \times \operatorname{curl} \boldsymbol{b}_{h}\right) \cdot \boldsymbol{k} d a=0 \text { for all } j \text { and } h .
$$

The phase constant $\alpha$ corresponding to each $\boldsymbol{b}_{j}$ is given by

$$
\alpha^{2}=\mu \epsilon \omega^{2}-\beta_{i}^{2},
$$

and $\boldsymbol{L}_{j}=\boldsymbol{b}_{j} \exp ( \pm j \alpha z)$ satisfies the vector wave equation

$$
\text { curl curl } \boldsymbol{L}_{j}=\mu \epsilon \omega^{2} \boldsymbol{L}_{j} .
$$

Utilizing the above properties of the normal modes, the field within a guide excited by an arbitrary current distribution will be determined by a method due to Bethe [4].

Using Gauss' Theorem, if $\boldsymbol{A}$ and $\boldsymbol{D}$ are two arbitrary vectors,

$$
\begin{aligned}
& \int_{S}[\boldsymbol{A} \times \operatorname{curl} \boldsymbol{D}-\boldsymbol{D} \times \operatorname{curl} \boldsymbol{A}] \cdot \boldsymbol{n} d a= \\
& \int_{V}(\boldsymbol{D} \cdot \nabla \times \nabla \times \boldsymbol{A}-\boldsymbol{A} \cdot \nabla \times \nabla \times \boldsymbol{D}) d \tau,
\end{aligned}
$$

where $\boldsymbol{n}$ is the outward drawn normal to $S$, enclosing the volume $V$ whose element is $d \tau$. If one lets $\boldsymbol{A}=\boldsymbol{E}$, the electric field which satisfies

$$
\text { curl } \operatorname{curl} \boldsymbol{E}-\mu \epsilon \omega^{2} \boldsymbol{E}=-j \omega \mu \boldsymbol{J},
$$

where $\boldsymbol{J}$ is the current density, and lets $\boldsymbol{D}$ be a solution of

$$
\nabla \times \nabla \times \boldsymbol{D}-\mu \epsilon \omega^{2} \boldsymbol{D}=0,
$$

then eq 98 becomes

$$
\int_{S}(\boldsymbol{E} \times \nabla \times \boldsymbol{D}-\boldsymbol{D} \times \nabla \times \boldsymbol{E}) \cdot \boldsymbol{n} d a=-\mu j \omega \int_{V} \boldsymbol{J} \cdot \boldsymbol{D} d \tau .
$$


Consider a current element within the guide at $z=z^{\prime}$, and take matched loads at the two planes, $z=0$ and $z=z_{1}$. Expanding the field $E$ in terms of the normal modes, let

$$
\left.\begin{array}{l}
\boldsymbol{E}=\sum_{j} A_{j} \boldsymbol{b}_{j} \exp \left(-j \alpha_{j} z\right) z>z^{\prime} \\
\boldsymbol{E}=\sum C_{j} \boldsymbol{b}_{j} \exp \left(+j \alpha_{j} z\right) z<z^{\prime}
\end{array}\right\}
$$

Since $\boldsymbol{E}$ is continuous at $z=z^{\prime}$, and since the $\boldsymbol{b}_{j}$ are orthogonal,

$$
A_{j} \exp \left(-j \alpha_{j} z^{\prime}\right)=C_{j} \exp \left(j \alpha_{j} z^{\prime}\right) .
$$

If one substitutes the value of $\boldsymbol{E}$ given by eq 101 in eq 100 , and lets $\boldsymbol{D}=\boldsymbol{b}_{j} \exp \left(-j \alpha_{j} z\right)$, and integrates the left side of eq 100 over the plane $z=0$, one gets

$$
\begin{aligned}
& -\int_{z=0} \sum_{h} C_{h}\left[\boldsymbol{b}_{h} \times \nabla \times\left(\boldsymbol{b}_{j} \exp \left(-j \alpha_{j} z\right)\right)\right. \\
& -\boldsymbol{b}_{j} \times \nabla \times\left(\boldsymbol{b}_{h} \exp \left(+j \alpha_{h} z\right)\right] \cdot \boldsymbol{k} d a,
\end{aligned}
$$

or,

$$
\begin{aligned}
& -\sum C_{h} \boldsymbol{J}\left[\boldsymbol{b}_{h} \times \nabla \times \boldsymbol{b}_{j}-j \alpha_{j} \boldsymbol{b}_{h} \times\left(\boldsymbol{k} \times \boldsymbol{b}_{j}\right.\right. \\
& \left.-\boldsymbol{b}_{j} \times \nabla \times \boldsymbol{b}_{h}-j \alpha_{h} \boldsymbol{b}_{j} \times\left(\boldsymbol{k} \times \boldsymbol{b}_{h}\right)\right] \cdot \boldsymbol{k} d a .
\end{aligned}
$$

Because of the orthogonality relation (eq 96), the above simplifies to

$-\sum_{h} C_{h}\left[-j \alpha_{j} \boldsymbol{\int} \boldsymbol{b}_{h} \cdot \boldsymbol{b}_{j} d a-j \alpha_{h} \boldsymbol{\int} \boldsymbol{b}_{j} \cdot \boldsymbol{b}_{h} d a=2 C_{j} \alpha_{j} j\right.$.

The surface integral over the plane $z=z_{1}$ vanishes, and one has, from eq 100 ,

$$
C_{j}=-\frac{\mu \omega}{2 \alpha_{j}} \int_{V} \boldsymbol{J} \cdot \boldsymbol{b}_{j} \exp \left(-j \alpha_{j} z^{\prime}\right) d \tau
$$

Substituting eq 103 and eq 102 in eq 101, one finally gets

$$
\boldsymbol{E}=-\frac{\mu \omega}{2} \sum_{j} \boldsymbol{b}_{j} \boldsymbol{J} \frac{\boldsymbol{J} \cdot \boldsymbol{b}_{j} \exp \left(-j \alpha_{j}\left|z^{\prime}-z\right|\right)}{\alpha_{j}} d \tau .
$$

\section{Appendix 2}

Let

$$
\begin{array}{r}
I=\int_{0}^{\infty} E^{2} e^{-\frac{E}{k T}}\left\{\frac{\cos \sigma \sqrt{E}}{\sigma \sqrt{E}}-\frac{\sin \sigma \sqrt{E}}{\sigma^{2} E}\right\}^{2} d E, \\
\left\{\frac{\cos x}{x}-\frac{\sin x}{x^{2}}\right\}^{2}=2 \sum_{j=1}^{\infty}(-1)^{j} \frac{(2 x)^{2 j-2}}{(2 j) !} \frac{(j-1)}{(j+1)}
\end{array}
$$

which may be verified by performing the square, expressing the result in trigonometric functions of $2 x$, and then substituting for each trigonometric function its corresponding infinite series and combining.

If we substitute, eq 105 in eq 104, we obtain

$I=(k T)^{3} \sum_{j=1}^{\infty}(-1)^{j} 2^{2 j-1}\left(\sigma^{2} k T\right)^{j-1} \frac{(j-1) \gamma(j+2)}{(2 j) !(j+1)}$,

where we have utilized the formula for the gamma function,

$$
\int_{0}^{\infty} x^{n-1} e^{-a x} d x=\frac{\gamma(n)}{a^{n}}
$$

Using the duplication formula [16] for the $\gamma$ function,

$$
(2 j) !=2 j \gamma(2 j)=\frac{2^{2 j}}{\sqrt{\pi}} \gamma(j+1) \gamma\left(j+\frac{1}{2}\right),
$$

eq 106 simplifies to

$$
I=\frac{(k T)^{3}}{2}\left\{\gamma\left(\frac{3}{2}\right) \sum_{j=0}^{\infty} \frac{\left(-\sigma^{2} k T\right)^{j}}{\gamma\left(j+\frac{3}{2}\right)}-\gamma\left(\frac{1}{2}\right) \sum_{j=0}^{\infty} \frac{\left(-\sigma^{2} k T\right)^{j}}{\gamma\left(j+\frac{1}{2}\right)} .\right.
$$

From MacRobert's definition of the confluent hypergeometric function, it follows that

$$
F(\alpha, \rho, w)=\frac{\gamma(\rho)}{\gamma(\alpha)} \sum_{n=0} \frac{w^{n} \gamma(\alpha+n)}{\gamma(n+1) \gamma(\rho+n)} .
$$

Accordingly, eq A 109 may be written

$$
I=\frac{(k T)^{3}}{2}\left\{-F\left(1, \frac{1}{2},-w\right)+F\left(1, \frac{3}{2},-w\right)\right\},
$$

where $w=\sigma^{2} k T$

However, since

$$
\begin{array}{r}
F(1, \rho-1,-w)=1-\frac{\gamma(\rho-1)}{\gamma(\rho)} w F(1, \rho,-w), \\
I=\frac{(k T)^{3}}{2}\left\{(1+2 w) F\left(1, \frac{3}{2},-w\right)-1\right\} .
\end{array}
$$

Finally since

$$
\begin{gathered}
F(\alpha, \rho, w)=e^{w} F(\rho-\alpha, \rho,-w), \\
I=\frac{(k T)^{3}}{2}\left\{(1+2 w) e^{-w} F\left(\frac{1}{2}, \frac{3}{2}, w\right)\right\}
\end{gathered}
$$

Washington, October 6, 1948. 